\title{
Direct characterization of protein diffusion and retention in polyacrylamide hydrogels using fluorescence confocal microscopy
}

\author{
M.R. Burnham, D. Szarowski, D.L. Martin, and J.N. Turner.
}

New York State Department of Health, Wadsworth Center for Labs and Research, New York State Department of Health, PO Box 509, Empire State Plaza, Albany, NY 12202

Hydrogels are useful materials in a wide variety of biomedical applications, including in vivo drug delivery, tissue engineering, and the development of artificial organs and other implantable devices such as contact lenses [1]. They are also used experimentally as templates for selective cell growth, protein and nucleic acid microarrays, and $\mathrm{pH}$ and/or enzymatic microsensors [2-6]. Functionalized hydrogels are gels that have biological entities, such as antibodies or enzymes, covalently linked to them. Typically, functionalized hydrogels are made in one of two ways: the first involves linking biological entities to one of the polymeric components of the hydrogel in a pre-polymerization step; polymerization then results in functional groups randomly distributed throughout the hydrogel. The second involves polymerizing a "bare" hydrogel (nothing attached), followed by a treatment of the gel to covalently link functional groups to it. Using this second approach, the distribution of protein within the hydrogel is not known, but will depend on the ability of various components of the conjugation scheme to diffuse into and react with the gel.

Thick hydrogel films (20 um thick) containing polyacrylamide, the disulfide-containing crosslinker bis (acryloyl) cystamine, and bis-acrylamide (6\% acrylamide; 98:1:1 molar ratio, respectively) were polymerized without protein. Biotin/streptavidin (SA) complexes were then conjugated to the hydrogel according to the reaction scheme outlined in Figure 1. Iodoacetyl-biotin was introduced into the hydrogel in step 2 either by bath application, or by microcontact printing to generate discrete, localized regions of biotinylated hydrogel. Fluorescein-conjugated SA (FITC-SA) was then introduced into the hydrogel by bath application. Confocal microscopy was used to visualize the location of SA, and characterize the extent of penetration of the protein into the hydrogel.

In the absence of biotin, there was very little binding of SA to the hydrogel, indicating that nonspecific binding or passive retention of SA was negligible in this system (Figure 2, panel A). In contrast, SA readily bound to biotinylated hydrogels (panel x,y). Confocal microscopy of these samples revealed a diffuse band of fluorescence corresponding to SA located at the top surface of the hydrogel (Figure 2B, panels $x, z$ and $y, z$ ). The thickness of this band, reflecting the depth of penetration of SA into the hydrogel, was calculated to be ca. $6 \mu \mathrm{m}$. This is in comparison to the overall thickness of the hydrogel, which was ca. $20 \mu \mathrm{m}$. When biotin was microcontact printed onto the surface of the hydrogel, we observed discreet regions of SA that corresponded to the stamp pattern (Figure 3, panel $\mathrm{x}, \mathrm{y}$ ). Again, there was negligible binding of SA to regions of the hydrogel that did not contain biotin. Confocal microscopy of these samples revealed that the depth of penetration of SA on a microcontact-printed region of the hydrogel was similar to that seen in the bath-applied samples (ca. $5 \mu \mathrm{m}$ ).

These results indicated that the location of SA in the hydrogel is limited to a small zone at the top of the hydrogel. This is true whether biotin is bath applied to the gel, or microcontact printed on the surface of the gel. This apparent restriction in protein access to the entire volume of hydrogel 
resulted in less than $50 \%$ of the total binding capacity of the gel being filled. These observations have implications for protein loading and maximizing the capacity of the hydrogel for proteins and other functional moieties.

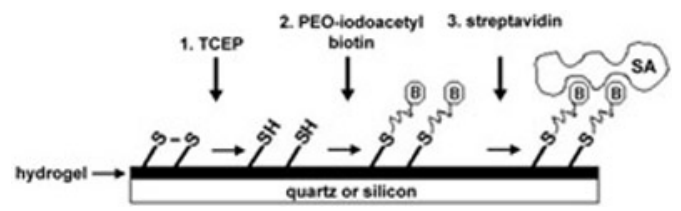

Figure 1: The reaction scheme for covalent linkage of streptavidin to polyacrylamide hydrogels. SS: disulfide bond of bis-CA; SH: reactive sulfhydryl group; B: biotin; SA: streptavidin.
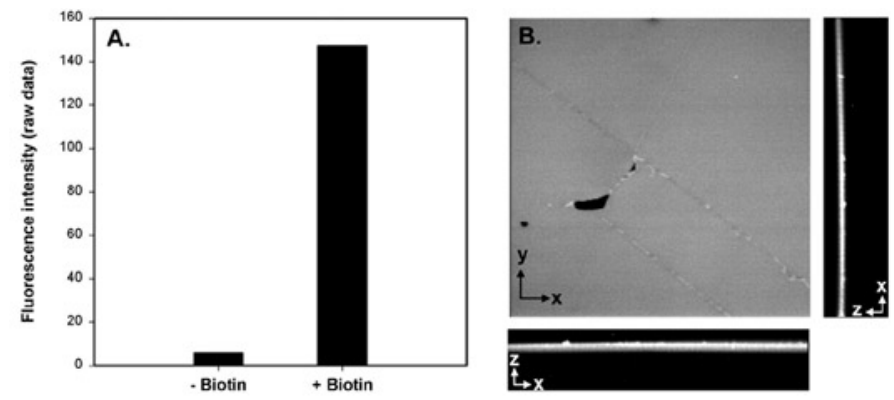

Figure 2: Panel A: Quantitation of FITC-SA binding to non-biotinylated and biotinylated hydrogels. Panel B: Confocal microscopy of hydrogels that received bath-applied biotin and FITC-SA. The large panel represents the maximum intensity $(\mathrm{x}, \mathrm{y})$ projection; the two smaller panels represent the $(\mathrm{x}, \mathrm{z})$ and $(\mathrm{y}, \mathrm{z})$ projections.

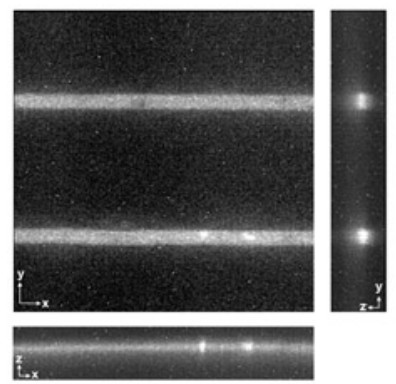

Figure 3: Confocal microscopy of hydrogels that received microcontact-printed biotin and FITC-SA. The total depth of scan was $50 \mu \mathrm{m}$, with $0.2 \mu \mathrm{m}$ step size. The large panel represents the maximum intensity $(\mathrm{x}, \mathrm{y})$ projection; the two smaller panels represent the $(\mathrm{x}, \mathrm{z})$ and $(\mathrm{y}, \mathrm{z})$ projections.

[1] J. Kopecek Euro. J. Pharm. Sci. 20 (2003) 1.

[2] H. Kobayashi et al., Curr. Eye Res. 10 (1991) 899.

[3] W.G. Koh et al., Biomed Microdev. 5 (2003) 11.

[4] M. Kuhner et al., Biophys. J. 67 (1994) 217.

[5] C. Jimenez et al., Analytica Chimica Acta. 351 (1997) 169.

[6] J.R. Retama et al., Biosensors and Bioelectronics. 20 (2004) 1111.

[7] This work was supported by the Nanobiotechnology Center (NBTC), an STC Program of the National Science Foundation (NSF) under Agreement No. ECS-9876771, and by a subcontract of grant 0325556 from the NSF (Principal Investigator, Stephen B. Wicker, Cornell University). 